\title{
Indacenodithiophene-benzothiadiazole organic field-effect transistors with gravure-printed semiconductor and dielectric on plastic
}

\begin{abstract}
Stuart G. Higgins*, Department of Physics and Centre for Plastic Electronics, Imperial College London, South Kensington Campus, London SW7 2AZ, UK Beinn V.O. Muir, Department of Physics and Centre for Plastic Electronics, Imperial College London, South Kensington Campus, London SW7 2AZ, UK; Department of Chemistry and Centre for Plastic Electronics, Imperial College London, South Kensington Campus, London SW7 2AZ, UK Martin Heeney, Department of Chemistry and Centre for Plastic Electronics, Imperial College London, South Kensington Campus, London SW7 2AZ, UK Alasdair J. Campbell, Department of Physics and Centre for Plastic Electronics, Imperial College London, South Kensington Campus, London SW7 2AZ, UK
\end{abstract}

Address all correspondence to Alasdair J. Campbell at alasdair.campbell@imperial.ac.uk

(Received 3 August 2015; accepted 9 September 2015)

\begin{abstract}
We demonstrate the gravure printing of a high-performance indacenodithiophene (IDT) copolymer, indacenodithiophene-benzothiadiazole $\left(\mathrm{C}_{16}\right.$ IDT-BT), onto self-aligned organic field-effect transistor architectures on flexible plastic substrates. We observed that the combination of a gravure-printed dielectric with gravure-printed semiconductor yielded devices with higher mean-effective mobility than devices manufactured using photolithographically patterned dielectric. Peak mobilities of $\mu=0.1 \mathrm{~cm}^{2} \mathrm{~V}^{-1} \mathrm{~s}^{-1}$ were measured, and exceed previous reports for non-printed $\mathrm{C}_{16}$ IDT-BT on non-flexible silicon substrates.
\end{abstract}

\section{Introduction}

The field of organic electronics allows us to develop new technologies such as flexible displays, radio frequency identification (RFID) tags, and intelligent packaging. ${ }^{[1-3]}$ The solubility of many organic semiconductors and dielectrics allows solution-based processes such as gravure, inkjet, and flexographic printing to be used ${ }^{[4]}$ introducing a manufacturing paradigm impossible with current silicon technology.

Gravure printing is well established as a manufacturing technique ${ }^{[5]}$ It facilitates high-speed, large-area printing and allows the patterning of multiple structures in parallel, providing a high-throughput solution. Gravure printing has already been demonstrated as viable for the manufacture of organic field-effect transistors (OFETs), ${ }^{[6-8]}$ organic light-emitting diodes (OLEDs), ${ }^{[9]}$ and other components vital for the development of flexible printed electronics. ${ }^{[10,11]}$ To fully realize these devices, organic semiconductors that are both compatible with printing and yield robust electrical characteristics are required.

One such series of semiconductors are polymers based upon indacenodithiophene (IDT). A variety of IDT copolymers have been reported yielding good performance in OFETs as well as organic photovoltaic devices. ${ }^{[12-17]}$ Good charge transport in these

* Current address: Optoelectronics Group, Cavendish Laboratory, University of Cambridge, Cambridge CB3 0HE, UK systems is attributed to the high rigidity of the polymer backbone, and correspondingly low conformational disorder. ${ }^{[12,18]}$

Recently Zhang et al. reported on an IDT copolymer, indacenodithiophene-benzothiadiazole $\left(\mathrm{C}_{16} \mathrm{IDT}-\mathrm{BT}\right),{ }^{[19]}$ with high field-effect mobilities that the authors found were relatively insensitive to processing conditions (tested using spin-coating in different laboratories). It is proposed that the dominant charge transport mechanism for the material is along the polymer backbone, which minimizes inter-chain hopping, and hence results in robust device characteristics. ${ }^{[20]}$ $\mathrm{C}_{16}$ IDT-BT is therefore of particular relevance in printed electronics, where it is desirable to minimize process variations caused during printing. Here we demonstrate how $\mathrm{C}_{16} \mathrm{IDT}-\mathrm{BT}$ can be successfully gravure printed onto a flexible selfaligned OFET architecture. We note that in our results device characteristics are sensitive to the dielectric deposition method used.

In this work, we manufacture self-aligned OFETs on a plastic substrate. Self-aligned architectures provide significant gains in device $\mathrm{AC}$ performance, thanks to minimized electrode overlaps. ${ }^{[21]}$ In addition, the layer-to-layer registration tolerance can be increased as the source and drain electrodes, as well as the effective channel length $(L)$, are defined by a prepatterned gate. Figure 1 illustrates a schematic of this architecture, along with the chemical structure of $\mathrm{C}_{16} \mathrm{IDT}-\mathrm{BT}$ and a photograph of a flexible substrate with gravure printed semiconductor. 
(a)
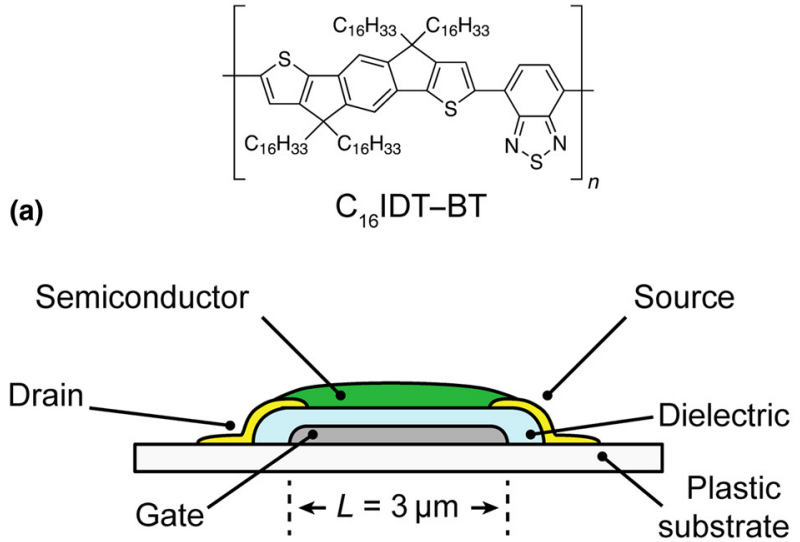

(b)

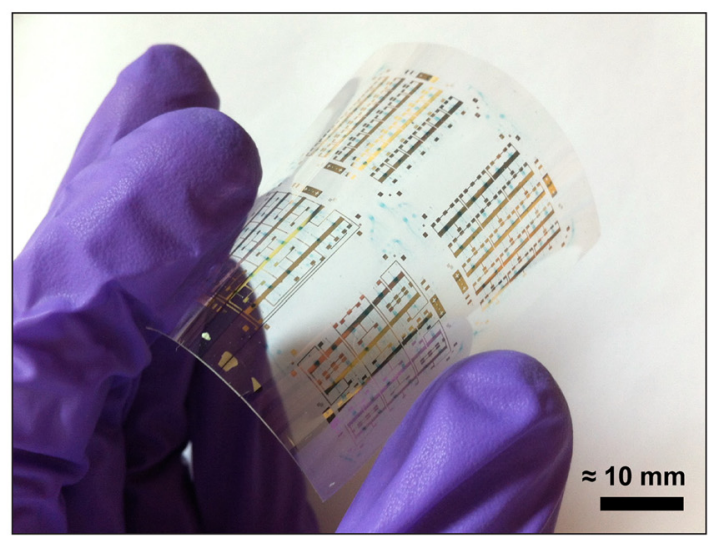

(c)

Figure 1. (a) Chemical structure of $C_{16}$ IDT-BT. (b) Schematic representation of the BGBC device architecture used in this work. (c) Photograph of the $C_{16}$ IDTBT gravure printed onto self-aligned transistor substrate.

\section{Experimental Gate patterning}

A $50 \mathrm{~nm}$ thick aluminum gate ( $\geq 99.9 \%$ purity, Testbourne Inc.) was evaporated onto a plastic foil, and patterned using photolithography (Microposit S1813, Rohm and Haas) via a mask aligner (MJB3, Süss).

\section{Dielectric patterning}

A proprietary dielectric system (GSID 938109-1, BASF SE), based upon a triacrylate cross-linking agent and high molecular weight poly(methyl methacrylate) (PMMA) viscosity modifier, was formulated into both photo-patternable and gravureprintable formulations, ${ }^{[22,23]}$ and patterned on top of the gate. For photo-patterned dielectric a formulation containing photo-initiator was spin-coated onto the substrate, soft-baked for $5 \mathrm{~min}$ at $115^{\circ} \mathrm{C}$ to drive off any remaining solvent, and selectively exposed in a mask aligner to partially cross-link an array of dielectric pads. Un-cross-linked material was removed by immersion in the formulation's solvent system and crosslinking completed by UV irradiation (ELC-500, Electro Lite Corporation) under nitrogen for $10 \mathrm{~min}$. For gravure-printed dielectric the formulation was filtered through a $0.45 \mu \mathrm{m}$ polytetrafluoroethylene (PTFE) filter onto an electrochemically etched gravure cliché (Goerz Gravurtechnik $\mathrm{GmbH}$ ), with a screen density of $l_{\mathrm{SD}}=250$ lines $/ \mathrm{cm}$.

\section{Self-aligned source-drain electrodes}

A bilayer lift-off process was used to define a nominal channel length of $L=3 \mu \mathrm{m}$ and width of $W=5000 \mu \mathrm{m}$, as well as interdigitated source-drain electrodes. We have previously reported full details of this process. ${ }^{[24]}$ In summary, a bilayer stack of $100 \mathrm{~nm}$ of lift-off resist (LOR1A, MicroChem Corporation) and $500 \mathrm{~nm}$ of photoresist (Microposit S1805 G2, Rohm and Haas) is exposed in a mask aligner. However, the substrate is inverted, so the UV light passes through both a standard photolithography mask and also the back of the substrate. Hence, the gate structure stops the resist stack immediately above the gate being exposed, defining the source-drain electrode spacing. The source-drain electrodes comprise a chromium adhesion layer $(\sim 5 \mathrm{~nm})$ and thermally evaporated gold $(\sim 50 \mathrm{~nm})$. Given the previous observations of the predominantly p-type nature of $\mathrm{C}_{16} \mathrm{IDT}-\mathrm{BT}$, substrates were immersed in a $5 \mathrm{mM}$ solution of pentafluorobenzenethiol (Sigma Aldrich) in ethanol for $10 \mathrm{~min}$ to form a self-assembled monolayer (SAM) on the gold electrodes to improve charge injection. ${ }^{[25]}$

\section{Gravure-printed semiconductor}

A $5 \mathrm{mg} / \mathrm{mL}$ ink formulation of $\mathrm{C}_{16}$ IDT-BT (Flexink Ltd) in chlorobenzene (purity $\geq 99.0 \%$, Merck) was prepared, with no further stirring required. The ink formulation was filtered through a $0.45 \mu \mathrm{m}$ PTFE filter onto the cliché inking edge, immediately prior to printing. Gravure printing was performed using a Labratester (Norbert Schläfli Maschinen), with a polyester doctor blade (E600/25/22, Esterlam International), and at a nominal nip pressure of $500 \mathrm{~N} / \mathrm{cm}^{2}$. The copper-based chromium-coated gravure clichés feature electrochemically etched cells in the printing surface, which define the pattern to be printed. Here we used gravure screen densities of $l_{\mathrm{SD}}=100$ lines $/ \mathrm{cm}$ and $l_{\mathrm{SD}}=250$ lines $/ \mathrm{cm}$, which correspond to a spacing of $\sim 100 \mu \mathrm{m}$ and $\sim 40 \mu \mathrm{m}$ between adjacent cells. In each print, four quadrants were patterned, each featuring a $5 \times 6$ array of semiconductor squares, each square having a nominal size of $2 \times 2 \mathrm{~mm}^{2}$. Printed substrates were annealed at $150{ }^{\circ} \mathrm{C}$ on a hotplate overnight in a glovebox. Electrical characterization was performed under nitrogen using a parameter analyzer (4156c, Agilent) and probe station (1260, Signatone).

\section{Results and discussion Gravure-printed $C_{16}$ IDT-BT}

The influence of both print speed and cliché screen density is shown in Fig. 2. It was found that at low print speeds $(s=$ $0.27 \mathrm{~m} / \mathrm{s}$ ) significant slurring (an increase in the size of a printed 


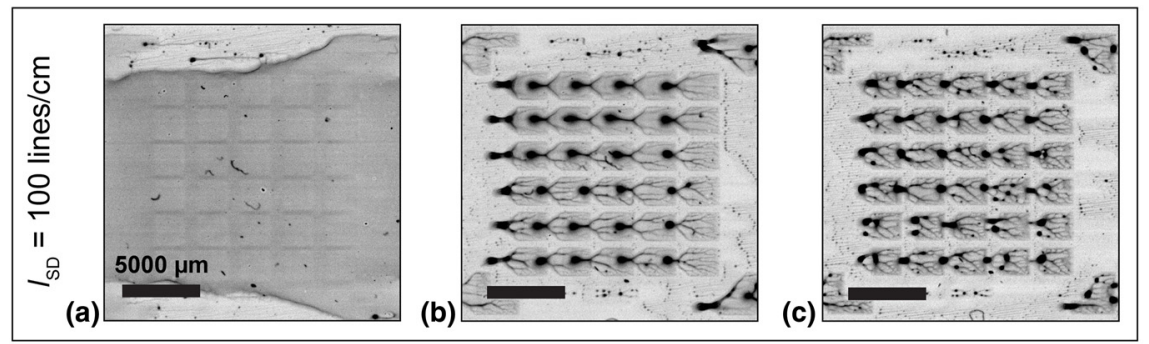

(g)
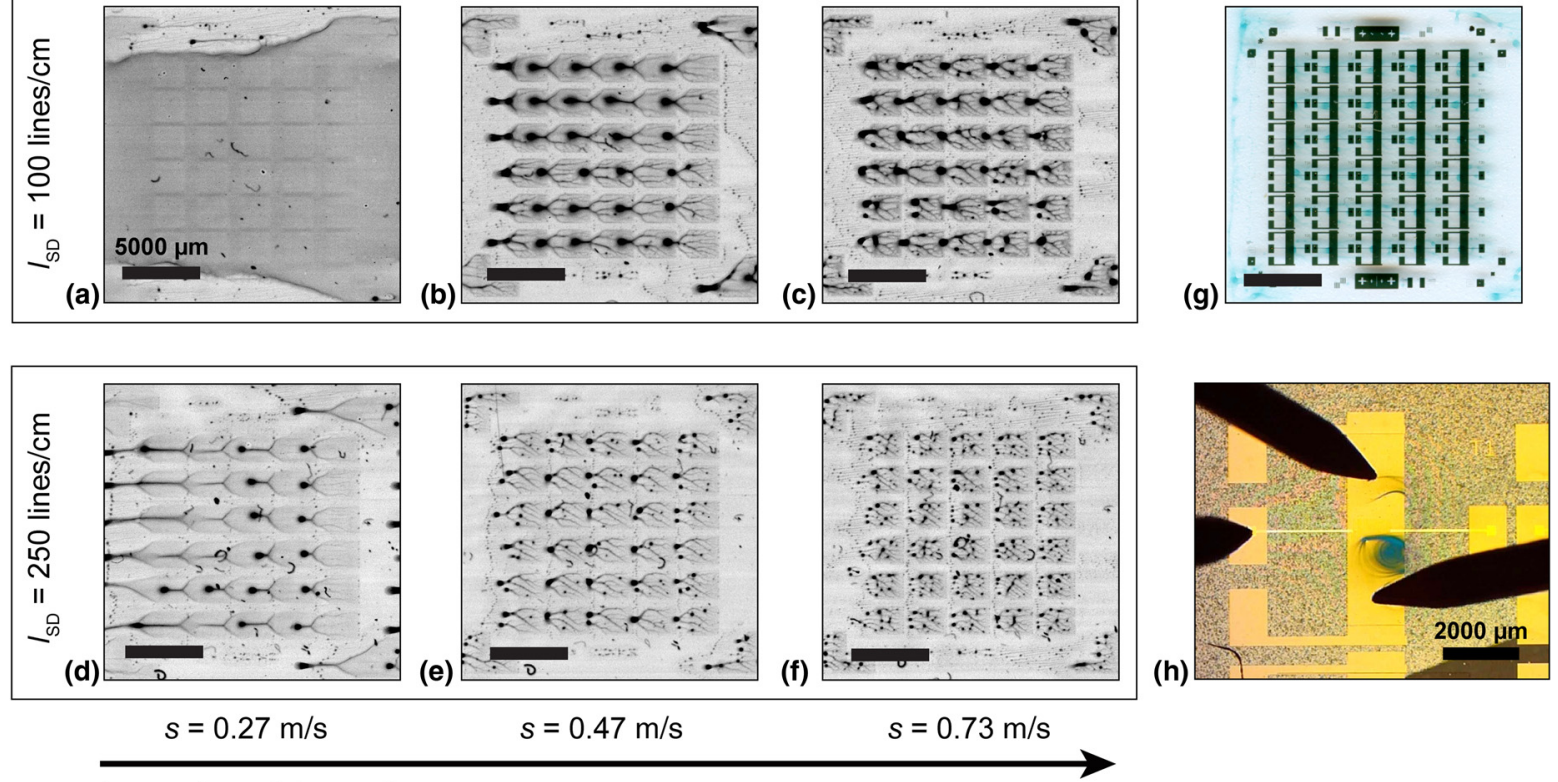

$s=0.47 \mathrm{~m} / \mathrm{s}$

(h)

Increasing print speed, $s$

Figure 2. Scanned images of gravure-printed $C_{16}$ IDT-BT on a plastic substrate, showing the impact of print speed and cliché screen density on print behavior. (a)-(c) $I_{S D}=100$ lines/cm, (d)-(f) $I_{S D}=250$ lines $/ \mathrm{cm}$. All scale bars equal for (a) $-(\mathrm{g})$. Images are shown in grayscale and contrast-enhanced for clarity. (g) Scanned image of the final gravure-printed semiconductor on top of the device architecture. (h) Optical micrograph of a single device under test.

feature compared with the corresponding size defined by the cliché) was observed. In the extreme case, this resulted in the coalescence of adjacent features [Fig. 2(a)] whereby the combination of low print speed and screen density results in an excessive volume of ink transfer onto the substrate. Such coalescence can potentially result in poor device isolation and high leakage currents. A screen density of $l_{\mathrm{SD}}=100$ lines $/ \mathrm{cm}$ was found to yield less macroscopic modulation of the semiconductor compared with $l_{\mathrm{SD}}=250$ lines $/ \mathrm{cm}$ [compare the modulation present in Figs. 2(c) and 2(f)]. This behavior occurs as a result of the corresponding decrease in cliché cell spacing from $\sim 100$ to $\sim 40 \mu \mathrm{m}$, resulting in a greater influence of the cell sidewalls. ${ }^{[23]}$ Less modulation is desirable, at the expense of the twodimensional (2D) semiconductor pad definition, as this minimizes variation of the semiconductor film thickness in the active device region. Film thickness variations can lead to incomplete semiconductor coverage in the channel region and differences in chain conformation and packing. These can impact channel conductivity, transport and trapping. Hence, a screen density of $l_{\mathrm{SD}}=100$ lines/cm was used to deposit the semiconductor in the devices presented below.

Figures 2(g) and 2(h) show a scanned image of the transistor array with gravure-printed semiconductor on top of the device architectures, as well as an optical micrograph of a single transistor under test. Thin-film semiconductors were very difficult to image in the final device architecture, with minimal contrast between the film and substrate; however, the excess of material in the center of the device in Fig. 2(h) suggests that some film modulation is present in the final devices, which agrees with the spread in device parameters shown in the electrical data discussed below (any variations in polymer coverage, chain conformation, and packing could impact the effective mobility, turn-on voltage, hysteresis, and off-current).

\section{$C_{16}$ IDT-BT organic field-effect transistors}

Figure 3 shows the electrical data obtained from devices with photo-patterned and gravure-printed dielectric. In the case of photo-patterned dielectric, p-type transistor behavior is observed with a peak-effective mobility of $\mu=0.02 \mathrm{~cm}^{2} \mathrm{~V}^{-1} \mathrm{~s}^{-1}$ at $V_{\mathrm{GS}}=V_{\mathrm{DS}}=-20 \mathrm{~V}$, and a median-effective mobility of $\mu \approx 10^{-4} \mathrm{~cm}^{2} \mathrm{~V}^{-1} \mathrm{~s}^{-1}$ (taking into account both forward and reverse sweeps). Here we refer to our extracted values as an effective mobility, as they are a convolution of both the intrinsic mobility of the material and also other effects, such as contact resistance which acts to lower the measured mobility. ${ }^{[26]}$ With this conservative estimate, these results compare well with the previous report of Zhang et al. ${ }^{[19]}$ of $\mu \approx 0.02 \mathrm{~cm}^{2} \mathrm{~V}^{-1} \mathrm{~s}^{-1}$ for $\mathrm{C}_{16}$ IDT-BT spin-coated on bottom-gate bottom-contact (BGBC) OFETs on a silicon substrate. Although lower than the performance reported for top-gate top-contact devices, our BGBC geometry facilitates self-alignment, with numerous other benefits. The lack of electrode overlap between gatedrain and gate-source electrodes results in very low leakage currents $(<0.1 \mathrm{nA}$ for photo-patterned devices). This lack of overlap reduces capacitive coupling between electrodes, increasing the operational speed of devices. ${ }^{[27]}$ The architecture also allows the semiconductor to be deposited last, minimizing the impact of subsequent processing.

For devices with a gravure-printed dielectric the peakeffective mobility increased to $\mu=0.1 \mathrm{~cm}^{2} \mathrm{~V}^{-1} \mathrm{~s}^{-1}$ at $V_{\mathrm{GS}}=$ 

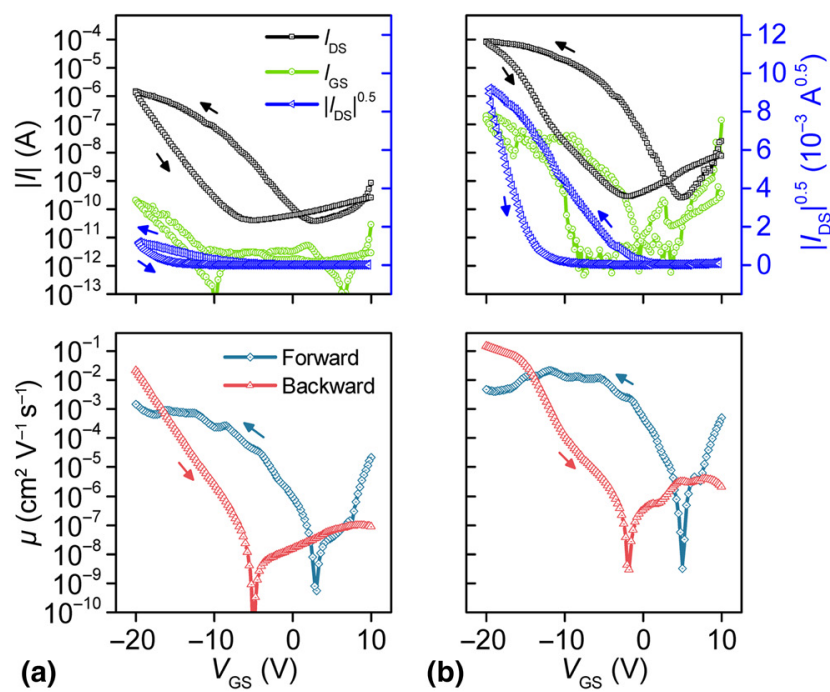

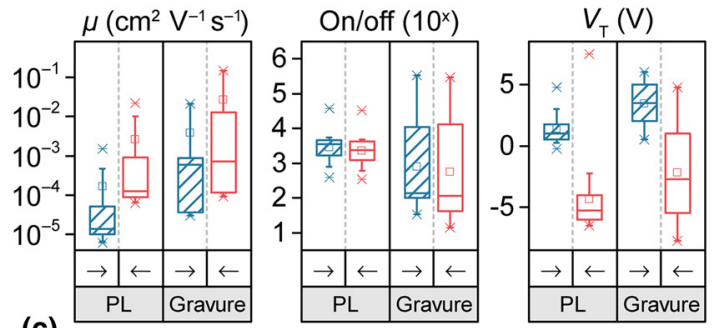

(c)

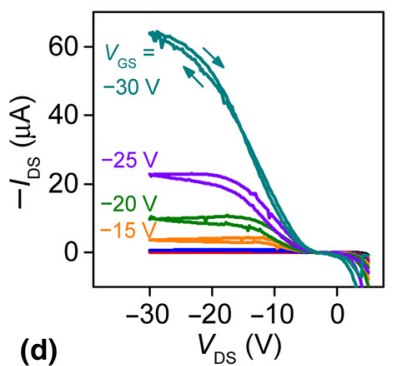

Figure 3. Electrical characterization data of gravure printed $\mathrm{C}_{16}$ IDT-BT OFETs on plastic. (a) Average transfer characteristics and extracted mobilities of 15 OFETs with photo-patterned dielectric. (b) Equivalent plots of average of six OFETs with gravure-printed dielectric. (c) Box plots summarizing the distribution of extracted figures of merit. Box represents 25th and 75th percentiles, horizontal line indicates median, square ( $\square$ ) the mean, whiskers the 10th and 90th percentiles, crosses $(x)$ the $\mathrm{min} / \mathrm{max}$ values. Values are extracted from forward $(\rightarrow)$ and reverse $(\leftarrow)$ sweeps. (d) Example of output characteristics obtained from the best-performing device (gravure-printed dielectric), corresponding to transfer characteristics shown in (b). Nominal device geometry is $W=5000$ and $L=3 \mu \mathrm{m}$.

$V_{\mathrm{DS}}=-20 \mathrm{~V}$, with a median-effective mobility of $\mu \approx 10^{-3}$ $\mathrm{cm}^{2} \mathrm{~V}^{-1} \mathrm{~s}^{-1}$. These results exceed previous reports and suggest that the dielectric layer formed by gravure printing facilitates better charge transport in $\mathrm{C}_{16}$ IDT-BT, at the expense of a slight increase in dielectric leakage. This leakage arises from the different film thicknesses of the photo-patterned and printed dielectric layers. Surface profilometry measurements of equivalent devices were used to determine film thicknesses of $t \approx$ $90 \mathrm{~nm}$ and $t \approx 170 \mathrm{~nm}$ for photo-patterned and printed dielectric layers, yielding specific capacitances $C^{\prime} \approx 33 \mathrm{nF} / \mathrm{cm}^{2}$ and $C^{\prime} \approx 17 \mathrm{nF} / \mathrm{cm}^{2}$, respectively. Similarly the yield for devices with gravure printed dielectric dropped to $18 \%$ ( $6 / 34$ devices), compared to $44 \%$ for photo-patterned $(15 / 34$ devices). This is due to lower breakdown voltages for the thinner dielectric. Hence, increasing the dielectric thickness either through modifying the print parameters, ${ }^{[23]}$ or overprinting, is desirable to reduce leakage and increase device robustness. ${ }^{[4]}$

It is important to consider the origin of the field-effect mobility enhancement observed in Fig. 3. Considering the standard OFET current-voltage relations, ${ }^{[28]}$ an approximate $50 \%$ thinning of the dielectric layer would yield an increase of 2 in the effective mobility, assuming all other parameters remain constant. However, the parameter extraction presented here is corrected for the increased specific capacitance of the thinner layer. The main difference between the two dielectric deposition methods, other than film thickness, is that the gravureprinted dielectric is thermally cross-linked, rather than photopatterned. The effective mobility of an OFET is strongly influenced by the dielectric-semiconductor interface, ${ }^{[29]}$ as are other behaviors such as hysteresis and the threshold voltage (discussed below). We hypothesize that the presence of residual photo-initiator in the photo-patterned dielectric degrades device characteristics, ${ }^{[30]}$ resulting in a lower-effective mobility compared with the gravure-printed devices.

Figure 3(c) summarizes the extracted figures of merit, illustrating the relative shift in mobility for devices with gravureprinted dielectric. Parameter extraction is performed on both the forward and reverse characterization sweeps to give a complete understanding of device behavior. Hysteresis is observed in both types of device, manifesting as a splitting of the threshold voltage on the forward and reverse sweeps, and indicating the presence of charge traps at the dielectric-semiconductor interface. We note that in previous reports using $\mathrm{C}_{16} \mathrm{IDT}-\mathrm{BT}$ a fluropolymer dielectric (CYTOP) was used, ${ }^{[19,20]}$ suggesting that dielectric dipolar disorder between the PMMA viscosity modifier in our dielectric ink and the semiconductor at the interface may increase charge trapping. ${ }^{[29]}$

The architecture presented here was designed to target highfrequency OFET switching, hence the use of a relatively shortchannel length $(L=3 \mu \mathrm{m})$. However, a consequence of this downscaling is an increase in the impact of contact resistance between the source-drain electrodes and semiconductor channel, as illustrated by the slight s-shaped slope in the output characteristics shown in Fig. 3(d). Further development of the contact interface (for example, through the exploration of different SAMs) is a route to minimizing this effect. ${ }^{[31-33]}$

\section{Conclusions}

Our findings show that $\mathrm{C}_{16} \mathrm{IDT}-\mathrm{BT}$ is a good candidate for high-performance printed electronics, yielding mobilities in 
the range of $\mu \approx 0.02-0.1 \mathrm{~cm}^{2} \mathrm{~V}^{-1} \mathrm{~s}^{-1}$ in our self-aligned BGBC architecture. Devices with gravure printed (rather than photo-patterned) dielectric yield higher mobilities, indicating an improved dielectric-semiconductor interface when both $\mathrm{C}_{16}$ IDT-BT and the dielectric are printed.

\section{Acknowledgments}

S.G.H. was supported by the Engineering and Physical Sciences Research Council (EPSRC) under grant number EP/P505550/1. Parts of this work have also been supported by the European Commission's 7th Framework Program (FP7/2007-2013) under grant agreement no. 247978 (POLARIC).

Open data statement: the underlying data supporting this publication is available at: http://dx.doi.org/10.5281/zenodo.30366

\section{References}

1. M.G. Kane: Organic thin-film transistors for flat-panel displays, in Organic Field-Effect Transistors, edited by Z. Bao and J. Locklin. (Taylor \& Francis Group, 2007) pp. 551.

2. T.-M. Lee, J.-H. Noh, C.H. Kim, J. Jo, and D.-S. Kim: Development of a gravure offset printing system for the printing electrodes of flat panel display. Thin Solid Films 518, 3355 (2010).

3. K.-J. Baeg, M. Caironi, and Y.-Y. Noh: Toward printed integrated circuits based on unipolar or ambipolar polymer semiconductors. Adv. Mater. 25, 4210 (2013).

4. M.M. Voigt, A. Guite, D.-Y. Chung, R.U.A. Khan, A.J. Campbell, D.D. C. Bradley, F. Meng, J.H.G. Steinke, S. Tierney, I. McCulloch, H. Penxten, L. Lutsen, O. Douheret, J. Manca, U. Brokmann, K. Sönnichsen, D. Hülsenberg, W. Bock, C. Barron, N. Blanckaert, S. Springer, J. Grupp, and A. Mosley: Polymer field-effect transistors fabricated by the sequential gravure printing of polythiophene, two insulator layers, and a metal ink gate. Adv. Funct. Mater. 20, 239 (2010).

5. H. Kipphan: Handbook of Print Media (Springer-Verlag, Heidelberg, 2001).

6. H. Kempa, M. Hambsch, K. Reuter, M. Stanel, G.C. Schmidt, B. Meier, and A.C. Hubler: Complementary ring oscillator exclusively prepared by means of gravure and flexographic printing. IEEE Trans. Electron Devices 58, 2765 (2011).

7. A. de la Fuente Vornbrock, D. Sung, H. Kang, R. Kitsomboonloha, and V. Subramanian: Fully gravure and ink-jet printed high speed pBTTT organic thin film transistors. Org. Electron. 11, 2037 (2010).

8. M. Hambsch, K. Reuter, M. Stanel, G. Schmidt, H. Kempa, U. Fügmann, U. Hahn, and A.C. Hübler: Uniformity of fully gravure printed organic field-effect transistors. Mater. Sci. Eng. B 170, 93 (2010).

9. D.-Y. Chung, J. Huang, D.D.C. Bradley, and A.J. Campbell: High performance, flexible polymer light-emitting diodes (PLEDs) with gravure contact printed hole injection and light emitting layers. Org. Electron. 11, 1088 (2010).

10. J. Noh and V. Subramanian: Fully gravure-printed D flip-flop on plastic foils using single-walled carbon-nanotube-based TFTs. IEEE Electron Device Lett. 32, 638 (2011).

11. M. Hambsch, K. Reuter, H. Kempa, and A.C. Hübler: Comparison of fully printed unipolar and complementary organic logic gates. Org. Electron. 13, 1989 (2012).

12.I. McCulloch, R.S. Ashraf, L. Biniek, H. Bronstein, C. Combe, J. E. Donaghey, D.I. James, C.B. Nielsen, B.C. Schroeder, and W. Zhang: Design of semiconducting indacenodithiophene polymers for high performance transistors and solar cells. Acc. Chem. Res. 45, 714 (2012).

13. M. Zhang, X. Guo, X. Wang, H. Wang, and Y. Li: Synthesis and photovoltaic properties of $D-A$ copolymers based on alkyl-substituted indacenodithiophene donor unit. Chem. Mater. 23, 4264 (2011).

14. Y.-C. Chen, C.-Y. Yu, Y.-L. Fan, L.-I. Hung, C.-P. Chen, and C. Ting: Low-bandgap conjugated polymer for high efficient photovoltaic applications. Chem. Commun. 46, 6503 (2010).
15.J.-S. Wu, Y.-J. Cheng, M. Dubosc, C.-H. Hsieh, C.-Y. Chang, and C.-S. Hsu: Donor-acceptor polymers based on multi-fused heptacyclic structures: synthesis, characterization and photovoltaic applications. Chem. Commun. 46, 3259 (2010).

16. Y. Zhang, J. Zou, H. Yip, K. Chen, D.F. Zeigler, Y. Sun, and A.K.-Y. Jen: Indacenodithiophene and quinoxaline-based conjugated polymers for highly efficient polymer solar cells. Chem. Mater. 23, 2289 (2011).

17. Y. Zhang, J. Zou, H. Yip, K. Chen, J.A. Davies, Y. Sun, and A.K.-Y. Jen: Synthesis, characterization, charge transport, and photovoltaic properties of dithienobenzoquinoxaline- and dithienobenzopyridopyrazine-based conjugated polymers. Macromolecules 44, 4752 (2011).

18. S. Holliday, J.E. Donaghey, and I. McCulloch: Advances in charge carrier mobilities of semiconducting polymers used in organic transistors. Chem. Mater. 26, 647 (2014).

19. X. Zhang, H. Bronstein, A.J. Kronemeijer, J. Smith, Y. Kim, R.J. Kline, L. J. Richter, T.D. Anthopoulos, H. Sirringhaus, K. Song, M. Heeney, W. Zhang, I. McCulloch, and D.M. DeLongchamp: Molecular origin of high field-effect mobility in an indacenodithiophene-benzothiadiazole copolymer. Nat. Commun. 4, 2238 (2013).

20. D. Venkateshvaran, M. Nikolka, A. Sadhanala, V. Lemaur, M. Zelazny, M. Kepa, M. Hurhangee, A.J. Kronemeijer, V. Pecunia, I. Nasrallah, I. Romanov, K. Broch, I. Mcculloch, D. Emin, Y. Olivier, J. Cornil, D. Beljonne, and H. Sirringhaus: Approaching disorder-free transport in high-mobility conjugated polymers. Nature 515, 384 (2014).

21. M. Caironi, Y.-Y. Noh, and H. Sirringhaus: Frequency operation of lowvoltage, solution-processed organic field-effect transistors. Semicond. Sci. Technol. 26, 034006 (2011).

22. N.L. Vaklev, R. Müller, B.V.O. Muir, D.T. James, R. Pretot, P. van der Schaaf, J. Genoe, J.-S. Kim, J.H.G. Steinke, and A.J. Campbell: Highperformance flexible bottom-gate organic field-effect transistors with gravure printed thin organic dielectric. Adv. Mater. Interfaces 1, 1300123 (2014).

23. S.G. Higgins, F.L. Boughey, R. Hills, J.H.G. Steinke, B.V.O. Muir, and A. J. Campbell: Quantitative analysis and optimization of gravure printed metal ink, dielectric, and organic semiconductor films. ACS Appl. Mater. Interfaces 7, 5045 (2015)

24. S.G. Higgins, B.V.O. Muir, J. Wade, J. Chen, B. Striedinger, H. Gold, B. Stadlober, M. Caironi, J.-S. Kim, J.H.G. Steinke, and A.J. Campbell: Self-aligned megahertz organic transistors solution-processed on plastic. Adv. Electron. Mater. 1, 1500024 (2015).

25. J.C. Love, L.A. Estroff, J.K. Kriebel, R.G. Nuzzo, and G.M. Whitesides: Self-assembled monolayers of thiolates on metals as a form of nanotechnology. Chem. Rev. 105, 1103 (2005).

26. F. Ante, D. Kälblein, T. Zaki, U. Zschieschang, K. Takimiya, M. Ikeda, T. Sekitani, T. Someya, J.N. Burghartz, K. Kern, and H. Klauk: Contact resistance and megahertz operation of aggressively scaled organic transistors. Small 8, 73 (2012).

27. U. Palfinger, C. Auner, H. Gold, A. Haase, J. Kraxner, T. Haber, M. Sezen, W. Grogger, G. Domann, G. Jakopic, J.R. Krenn, and B. Stadlober: Fabrication of $\mathrm{n}$ - and $\mathrm{p}$-type organic thin film transistors with minimized gate overlaps by self-aligned nanoimprinting. Adv. Mater. 22, 5115 (2010)

28. G. Horowitz: Organic field-effect transistors. Adv. Mater. 10, 365 (1998).

29. H. Sirringhaus: Device physics of solution-processed organic field-effect transistors. Adv. Mater. 17, 2411 (2005)

30. A. Petritz, A. Wolfberger, A. Fian, J.R. Krenn, T. Griesser, and B. Stadlober: High performance p-type organic thin film transistors with an intrinsically photopatternable, ultrathin polymer dielectric layer. Org. Electron. 14, 3070 (2013).

31. Y. Noh, N. Zhao, M. Caironi, and H. Sirringhaus: Downscaling of selfaligned, all-printed polymer thin-film transistors. Nat. Nanotechnol. 2, 784 (2007).

32. D. Khim, K.-J. Baeg, M. Caironi, C. Liu, Y. Xu, D.-Y. Kim, and Y.-Y. Noh: Control of ambipolar and unipolar transport in organic transistors by selective inkjet-printed chemical doping for high performance complementary circuits. Adv. Funct. Mater. 24, 6252 (2014).

33. D.Y. Chung, D.S. Leem, D.D.C. Bradley, and A.J. Campbell: Flexible multilayer inverted polymer light-emitting diodes with a gravure contact printed $\mathrm{Cs}_{2} \mathrm{CO}_{3}$ electron injection layer. Appl. Phys. Lett. 98, 2009 (2011). 